\title{
Avulsions dentaires chez les patients cardiaques traités par anticoagulants : résultats d'un essai thérapeutique acénocoumarol versus héparine calcique
}

\author{
Dental extractions in cardiac patients treated with anticoagulants: results of a \\ therapeutic trial of acenocoumarol versus heparin calcium
}

\author{
FADILA DAÏMELLAH ${ }^{1}$, MOHAND-SAÏD ISSAD', HAMID BOUKAÏS², WACILA ZERROUKI², MOHAMED BERKANE², \\ SALÉHA LEHACHI' ${ }^{1}$, ZAKIA BENNOUI ${ }^{1}$, SAIIDA KHELLIL ${ }^{1}$, DJOHAR HANNOUN ${ }^{3}$, SAMIRA ABROUK ${ }^{3}$
}

\section{RÉSUMÉ}

Background - En chirurgie buccale, la prise en charge des cardiaques sous anticoagulants est controversée. Objectif - Comparer l'incidence des complications hémorragiques des avulsions dentaires sous acénocoumarol avec un International Normalized Ratio la veille de l'acte entre 2.00 - 4.50, versus le relais par l'héparine calcique, en utilisant dans tous les cas des mesures locales d'hémostase.

Matériel et méthodes - Essai thérapeutique randomisé, contrôlé, en aveugle, monocentrique, réalisé entre avril 2002 et mars 2005. Les patients ont été hospitalisés en cardiologie au moins 48 heures avant les extractions dentaires. L'analyse a porté sur 135 patients dans le groupe acénocoumarol, 133 dans le groupe héparine.

Critère de jugement - Incidence des saignements post-opératoires nécessitant l'application de mesures d'hémostase locale, et de l'hématome buccal

Résultats - Dans chacun des groupes acénocoumarol et héparine calcique, le nombre des séances d'extractions était respectivement de 229 et 208 , le nombre de dents extraites / malade / séance étant respectivement de $1.96 \pm 0.23$ et $1.92 \pm 0.22(p=0.80)$. Le jour de l'acte, l'International Normalized Ratio était de $3.40 \pm 0.16$ dans le groupe acénocoumarol, le rapport temps de Céphaline Activé malade / temps de Céphaline Activé témoin de $1.45 \pm 0.10$ dans le groupe héparine. L'incidence du saignement a été plus élevée après le relais par l'héparine : $15.8 \%$ contre $6.7 \%, p=0.02$, avec un risque relatif de 2.40 . Le saignement est survenu entre $\mathrm{J} 0$ - J6. Tous les cas d'hémorragie ont été traités uniquement par les mesures locales d'hémostase.

Conclusion - Les résultats de ce premier essai thérapeutique comparant la poursuite de l'acénocoumarol au relais par l'héparine calcique en chirurgie dentaire ont des conséquences cliniques et économiques très importantes. Ils montrent que le rapport bénéfice-risque est plutôt en faveur de la poursuite de l'acénocoumarol et la réalisation des avulsions dentaires avec un International Normalized Ratio pré-opératoire $\leq 4.50$. (Med Buccale Chir Buccale 2009 ; 15: 63-74).

mots clés: anticoagulants, antivitamine K, héparine, extraction dentaire, hémostase locale en chirurgie buccodentaire, hémorragie post-opératoire en chirurgie bucco-dentaire

1. Service de Cardiologie, CHU Béni Messous

2. Service de Pathologie et Chirurgie buccale CHU Béni Messous

3. Institut National de Santé Publique (INSP) Alger

Demande de tirés à part:

Fadila Daïmellah Service de Cardiologie CHU Béni Messous Alger Algérie daimfadila@yahoo.fr 


\section{SUMMARY}

Background - In dental surgery, the care of patients treated with anticoagulation has been the subject of controversy.

Aim - Comparing the incidence of bleeding complications after dental extractions in patients treated with acenocoumarol and with an International Normalized Ratio (INR) the day before the act between 2.00 - 4.50, versus patients covered with heparin calcium after stopping acenocoumarol, using in all cases local hemostatic measures: oxycellulose mesh $\left(\right.$ surgice $\left.{ }^{\star}\right)+$ sutures + compression gutter.

Material and methods - Randomized blind therapeutic trial, controlled, monocentric, realized between april 2002 and marsh 2005. All patients were hospitalized in cardiology service for at less 48 hours before the procedure. Analysis concerned 135 patients in the acenocoumarol group and 133 in the heparin calcium one.

Endpoint - Incidence of all bleedings requiring the use of local hemostatic measures and of buccal hematoma, after dental extractions.

Results - In each group acenocoumarol and heparin calcium, the extractions sessions number was respectively 229 and 208, the extracted teeth number/patient/session was respectively $1.96 \pm 0.23$ and $1.92 \pm 0.22(p=0.80)$. The day of intervention, INR was $3.40 \pm 0.16$ in the acenocoumarol group, the ratio of the patient's activated partial thromboplastin time $(\mathrm{aPTT})$ to the mean control $\mathrm{aPTT}$ was $1.45 \pm 0.10$ in the heparin group. Bleeding incidence was higher after heparin therapy coverage : $15.8 \%$ against $6,7 \%(p=0,02)$, with a relative risk of 2,40. Bleeding occurred between D0-D6. All bleeding cases have been treated by hemostasis local measures.

Conclusion - Results of this first therapeutic trial comparing acenocoumarol continuation to the heparin therapy coverage in buccal surgery have very important clinical and economic consequences. They show that the ratio

médecine buccale chirurgie buccale

VOL. $15, \mathrm{~N}^{\circ} 2$ 2009

page 64 benefit-risk is rather in favour of acenocoumarol continuation and realization of dental avulsions with a pre-operative INR $\leq 4$, 50. (Med Buccale Chir Buccale 2009 ; 15: 63-74).

key words: anticoagulants, antivitamin K, heparin, dental extraction, local hemostasis in oral surgery, postoperative bleeding in oral surgery

\section{ABREVIATIONS}

AVK : antivitamine $K$

INR : international normalized ratio

$R R$ : risque relatif

TCA : temps de Céphaline Activé
TCAm / TCAt : temps de céphaline activé malade / temps de céphaline activé témoin

$\mathrm{TIH}$ : thrombopénie induite par l'héparine
La réalisation d'extractions dentaires chez les patients sous AVK est à l'origine de difficultés fréquentes, en raison du risque hémorragique accru. Cependant, pour diminuer le risque de saignement, leur arrêt ou leur diminution, même temporaire, expose à des complications thromboemboliques potentiellement graves voire mortelles [1].

Les protocoles proposés vont du relais pré-opératoire par l'héparine, à la diminution de la posologie des AVK, jusqu'à leur arrêt quelques jours avant l'intervention chirurgicale [2,3]. L'alternative qui est de plus en plus évoquée propose de ne pas modifier les AVK en utilisant des mesures d'hémostase locale [2, 3].
L'absence d'étude randomisée comparant la poursuite des AVK au relais par l'héparine en chirurgie buccale [3] a mené à la réalisation d'un essai thérapeutique randomisé, contrôlé, en aveugle, monocentrique, dans le Service de Cardiologie du CHU Béni Messous situé dans la région Ouest d'Alger, en collaboration avec le service de Pathologie et Chirurgie buccale du même centre hospitalier. Ce travail s'est fondé sur l'hypothèse que la réalisation des extractions dentaires sous acénocoumarol (seul AVK actuellement commercialisé en Algérie) aux doses thérapeutiques ne majorait pas le risque hémorragique par rapport au relais par l'héparine calcique lorsque les mesures d'hémostase locale sont appliquées. 
L'objectif de cet essai était de comparer l'incidence du saignement des avulsions dentaires sous acénocoumarol avec un INR la veille de l'acte entre 2.00 et 4.50 , versus le relais par l'héparine calcique, en appliquant dans tous les cas des mesures locales d'hémostase.

\section{MATÉRIEL ET MÉTHODES}

\section{Participants}

Les malades ont été recrutés entre avril 2002 et mars 2005. Ils étaient traités par acénocoumarol au long cours et nécessitaient une ou de plusieurs extractions dentaires simples ou chirurgicales, en une ou plusieurs séances. Ont été exclus les patients présentant une infection dentaire, une hypertension artérielle non stabilisée (pression artérielle $\geq 160 / 95 \mathrm{~mm} \mathrm{Hg}$ ), un taux de plaquettes $<100000 / \mathrm{mm}^{3}$, des antécédents d'allergie à l'héparine et de THI, un infarctus du myocarde de moins de 3 semaines, un angor instable et ceux n'ayant pas donné leur consentement éclairé. L'étude a été réalisée en double aveugle par rapport aux chirurgiens dentistes, en simple aveugle par rapport aux cardiologues. Tous les sujets éligibles ont été hospitalisés dans le Service de Cardiologie au moins 48 heures avant la première séance d'extraction. La randomisation a été réalisée en cardiologie par un cardiologue participant à l'étude. Le code de randomisation a été établi avec une table de nombre au hasard randomisant par blocs de six. Les patients sont restés hospitalisés jusqu'à l'ablation des fils de suture.

Cet essai thérapeutique a été approuvé par le Comité d'éthique.

\section{Schéma de l'étude Traitement anticoagulant}

Pour l'acénocoumarol, c'est le Sintrom ${ }^{\circledR}$ (Novartis Pharma S.A), comprimés blancs $4 \mathrm{mg}$, quadrisécables, qui a été utilisé. La dose journalière a été répartie en 2 prises : 8 heures, 20 heures. Durant cette étude, nous avons été confrontés au problème de disponibilité de l'héparine calcique, situation qui ne pouvait être prévue lors de l'élaboration du protocole. Pendant l'année 2002, nous avons employé la Calciparine ${ }^{\circledR}$ (Léo France, seringues pré-remplies pour injections sous-cutanées). A partir de l'année 2003, le laboratoire Léo France ne fabriquant plus ce produit, nous avons été contraint d'utiliser Ecabil ${ }^{\circledR}$ (Biologici Italia, ampoules pour injections sous-cutanées), seule héparine calcique disponible à l'hôpital cette année là, remplacée en 2004 et 2005 par la Calciparine ${ }^{\circledR}$ (Choay Sanofi Winthrop France, seringues préremplies pour injections sous-cutanées).

Dans le groupe acénocoumarol, le traitement AVK n'a pas été modifié. Dans le groupe héparine calcique, l'acénocoumarol a été arrêté 48 heures avant l'intervention chirurgicale. Le protocole et la surveillance biologique des anticoagulants figurent dans les Tab. 1 et 2.

\section{Extractions dentaires et mesures d'hé- mostase locale}

Après désinfection du site à la bétadine, une anesthésie locale para-apicale (vestibulaire et palatine ou linguale) et, en cas de nécessité, intra-ligamentaire mandibulaire ont été pratiquées ; aucune anesthésie tronculaire n'a été réalisée en raison du son risque hémorragique. Toutes les avulsions ont été réalisées sous anesthésie avec vasoconstricteur : Scandicaïne ${ }^{\circledR} 2 \%$ noradrénaline (Septodont France, contenant 35 mg de chlorhydrate de mépivacaïne, $0.036 \mathrm{mg}$ de tartrate de noradrénaline). Le nombre de cartouches anesthésiques utilisé par malade a été inférieur à 4 par séance. Après extraction de la dent, révision de l'alvéole et compression bidigitale de quelques minutes, l'alvéole a été comblé avec une gaze de cellulose régénérée oxydée (Surgicel ${ }^{\circledR}$ Ethicon, Johnson \& Johnson). Puis, des points de sutures ont été effectués à l'aide de fil non résorbable et une gouttière hémostatique mise en place. Selon les conditions de rétention, deux types de gouttières ont été utilisés. Dans la majorité des cas, il s'agissait de gouttières extemporanées réalisées à partir d'une pâte siliconée $\left(\right.$ Optosi $\left.{ }^{\circledR}\right)$ renforcée par un élastomère de synthèse (Xantopren ${ }^{\circledR}$ ou Tewesil $\left.{ }^{\circledR}\right)$ : préparation facile, immédiate, utilisant des matériaux peu onéreux et toujours disponibles dans les cabinets dentaires. En l'absence d'appui, des gouttières en résine, renforcées le jour de l'extraction avec un élastomère de synthèse $\left(\right.$ Xantopren ${ }^{\circledR}$ ou Tewesil ${ }^{\circledR}$ ) ont été utilisées. médecine

buccale

chirurgie

buccale

VOL. $15, \mathrm{~N}^{\circ} 2$ 2009

page 65 
Tableau 1 : Protocole et surveillance du traitement dans le groupe acénocoumarol.

Protocol and treatment observation in the acenocoumarol group.

INR

Protocole

$J-1$ *

2.00 - 4.50 Acénocoumarol $\rightarrow$ même dose. Extraction réalisée le lendemain.

$<2.00$ Acénocoumarol $\rightarrow \uparrow$ la dose de $1 \mathrm{mg}$. Report de la séance d'extraction.

Extraction réalisée le lendemain de l'obtention d'un INR entre $2.00-4.50$.

$4.51-4.99$ Acénocoumarol $\rightarrow \downarrow$ la dose de $1 \mathrm{mg}$. Report de la séance d'extraction.

Extraction réalisée le lendemain de l'obtention d'un INR entre $2.00-4.50$.

$\geq 5.00 \quad$ Acénocoumarol $\rightarrow$ arrêt. Report de la séance d'extraction.

Ré-introduction de l'acénocoumarol dès que I'INR était $<3.00$ à la dose de $0.5 \mathrm{mg} 2$ fois par jour.

Extraction réalisée le lendemain de l'obtention d'un INR entre $2.00-4.50$.

$\mathrm{J} 0^{\text {** }}$

$2.00-3.00$ Risque thromboembolique modéré :

acénocoumarol $\rightarrow$ continuer même dose. Contrôle INR, TCA 48 heures plus tard.

Risque thromboembolique élevé :

acénocoumarol $\rightarrow \uparrow$ la dose de $1 \mathrm{mg}$. Contrôle INR, TCA 48 heures plus tard.

médecine

buccale

chirurgie

buccale

VOL. $15, \mathrm{~N}^{\circ} 2$ 2009

page 66

$3.01-4.50$ Risque thromboembolique modéré :

acénocoumarol $\rightarrow \downarrow$ la dose de $1 \mathrm{mg}$. Contrôle INR, TCA 48 heures plus tard.

Risque thromboembolique élevé :

acénocoumarol $\rightarrow$ continuer même dose. Contrôle INR, TCA 48 heures plus tard.

$<2.00$

Acénocoumarol $\rightarrow \uparrow$ la dose de $1 \mathrm{mg}$. Contrôle INR, TCA 48 heures plus tard.

$>4.50$ Acénocoumarol $\rightarrow$ arrêt. Contrôle INR, TCA 24 heures plus tard. Introduction de l'acénocoumarol

à la dose de $0.5 \mathrm{mg} 2$ fois par jour dès que l'INR était $<3.00$.

INR : Analyse semi-automatique par méthode chronométrique (ISI des réactifs : 1.0 - 2.8).

TCA : Analyse semi-automatique par méthode chronométrique.

* À midi, réalisation systématique de l'INR et du TCA.

** À 8 heures du matin, avant la séance d'extraction, réalisation systématique de l'InternalNR et du TCA, mais les résultats n'étaient connus que l'après-midi, c'est-à-dire après l'intervention chirurgicale.

Des conseils visant à diminuer le risque hémorragique ont été prodigués : éviction du tabac, bains de bouche les 24 premières heures, alimentation liquide ou semi-liquide, tiède ou froide, et brossage limité aux dents de voisinage.

\section{Traitements associés}

Pour la prophylaxie de l'endocardite infectieuse, ce sont les recommandations de la conférence de consensus de la Société de Pathologie Infectieuse de Langue Française de 1992 qui ont été appliquées [2]. Pour le traitement antalgique, c'est le paracétamol qui a été prescrit. Chez les patients qui étaient en plus sous antiagrégants plaquettaires, cette thérapeutique a été arrêtée une semaine avant les extractions dentaires et réintroduite après ablation des fils de suture.

\section{Critère d'évaluation}

II s'agissait de la survenue, au-delà de la deuxième heure suivant l'extraction dentaire, d'un saignement en jet ou en nappe, ou de la formation d'un caillot exubérant saignant à sa base (Fig. 1), ou encore de l'apparition d'un hématome buccal ; ce délai a été pris afin de ne pas prendre en compte les saignements secondaires à la vasodilatation survenant après l'arrêt de l'action du vasoconstricteur Ont été exclus, les suinte- 
Tableau 2 : Protocole et surveillance du traitement dans le groupe héparine calcique.

Protocol and treatment observation in the heparin group.

\section{Protocole et surveillance de l'héparine calcique}

\begin{tabular}{|c|c|}
\hline $\mathbf{J}-2$ & $\begin{array}{l}\text { Acénocoumarol } \rightarrow \text { arrêt. } \\
\text { Héparine } \rightarrow \text { bolus intra-veineux d'héparine sodique (50 Ul/Kg), suivi d'une injection sous-cutanée } \\
\text { d'héparine calcique } 3 \text { fois/jour : } 8 \mathrm{~h}, 16 \mathrm{~h} \text {, minuit (dose probatoire }=500 \mathrm{Ul} / \mathrm{Kg} / 24 \mathrm{~h} \text { ). } \\
\text { Ajustement des doses d'héparine calcique selon les résultats du rapport TCAm / TCAt effectué } 4 \mathrm{~h} \\
\text { après l'injection : } \\
-<1.5: \uparrow \text { la dose de } 0.05 \mathrm{ml} \text {; } \\
-1.5-2.0: \text { maintien de la même dose ; } \\
-2.0-3.0: \downarrow \text { la dose de } 0.05 \mathrm{ml} \text {. Contrôle TCA } 4 \text { heures plus tard ; } \\
->3.0: \text { sauter l'injection suivante, puis réintroduction de l'héparine calcique en diminuant la } \\
\text { dose de } 0.05 \mathrm{ml} \text {. }\end{array}$ \\
\hline$J-1^{*}$ & Héparine calcique prescrite et surveillée selon le protocole ci-dessus. \\
\hline $\mathbf{J} \mathbf{O}^{\text {**}}$ & $\begin{array}{l}\text { À } 8 \text { heures du matin, pas d'injection d'héparine calcique. Réintroduction de l'héparine calcique } \\
\text { après l'intervention, à partir de } 16 \text { heures. Ajustement des doses d'héparine et surveillance selon } \\
\text { le protocole ci-dessus. }\end{array}$ \\
\hline$>\mathbf{J} 0$ & $\begin{array}{l}\text { Héparine calcique prescrite et surveillée selon le protocole ci-dessus. Quand } 2 \text { TCA consécutifs } \\
\text { étaient entre } 1.5-2.0 \text {, les contrôles ont été plus espacés (toutes les } 24 \text { h). Surveillance } \\
\text { hebdomadaire du taux de plaquettes. }\end{array}$ \\
\hline
\end{tabular}

Relais héparine calcique $\rightarrow$ acénocoumarol

\begin{tabular}{cl}
\hline & Introduction de l'acénocoumarol à partir de 20 heures. Pour la dose probatoire \\
ont été pris en compte, les doses moyennes d'acénocoumarol mentionnées dans les carnets de \\
surveillance et l'INR pré-opératoire à J 0 :
\end{tabular}

INR : Analyse semi-automatique par méthode chronométrique (ISI des réactifs : 1.0 - 2.8).

TCA : Analyse semi-automatique par méthode chronométrique.

TCAm/TCAt : Temps de Céphaline Activé malade / Temps de Céphaline Activé témoin

* À midi, réalisation systématique de l'INR et du TCA

** À 8 heures du matin, avant la séance d'extraction, réalisation systématique de INR et du TCA, mais les résultats n'étaient connus que l'après-midi, c'est-à-dire après l'intervention chirurgicale.

${ }^{\star * \star}$ Ce protocole a été appliqué pour réduire la durée du relais et le risque de survenue de la thrombopénie induite par l'héparine.

ments avec crachats sanguinolants s'arrêtant au bout d'une vingtaine de minutes à l'aide d'une compresse mordue par le patient. Le suintement correspond à l'exsudation physiologique de la plaie de réparation.

\section{Suivi des patients}

Des examens de contrôle ont été réalisés systématiquement à $\mathrm{J} 1, \mathrm{~J} 3$ ou $\mathrm{J} 4$ pour le retrait de la gouttière, $\mathrm{J} 7$ ou $\mathrm{J} 8$ pour l'ablation des fils. Devant un saignement postopératoire, les patients devaient mordre sur une compresse appliquée sur le site opératoire. Si le saignement persistait au-delà de vingt minutes de compression, un INR ou un TCA étaient demandés afin d'adapter, au besoin, les doses du traitement anticoagulant selon les protocoles figurant dans les Tab. 1 et 3 , et les malades étaient ré-adressés en chirurgie buccale. Au plan local, devant un caillot exubérant, il a été procédé à son curetage sous anesthésie locale. Les diverses étapes de la procédure hémostatique ont été reprises, la gouttière réajus- médecine

buccale chirurgie buccale

VOL. $15, \mathrm{~N}^{\circ} 2$ 2009

page 67 

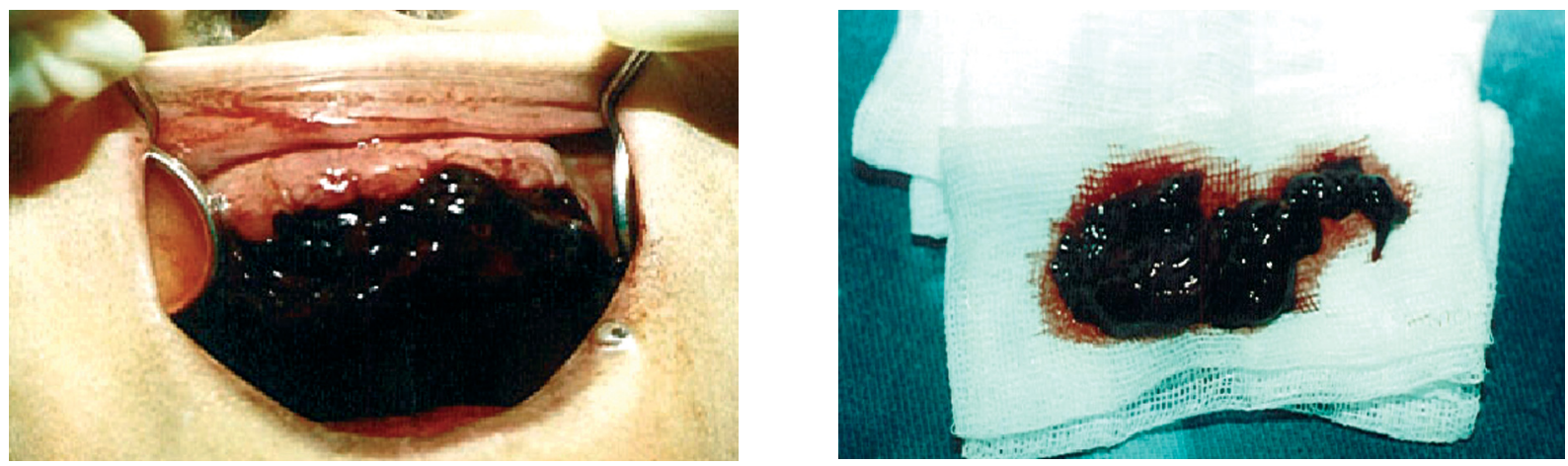

Figure 1 : Aspect du caillot exubérant.

Large clot.

Tableau 3 : Ajustement des doses d'héparine calcique lors de saignement. Adjustment of heparin calcium doses in bleeding dental cases.

médecine

buccale

chirurgie

buccale

VOL. $15, \mathrm{~N}^{\circ} 2$

2009

page 68

\begin{tabular}{rll}
\hline TCAm/TCAt & \multicolumn{1}{c}{ Protocole } \\
\hline$<1.5$ & A. $\uparrow$ la dose d'héparine de $0.05 \mathrm{ml}$. Contrôle TCA 4 heures plus tard \\
$1.5-$ & $\begin{array}{l}\text { B. Maintenir la même dose d'héparine. Contrôle TCA } 4 \text { heures plus tard } \\
\text { C. Sauter l'injection suivante puis, réintroduction de l'héparine en diminuant la dose de } \\
\end{array}$ \\
& $\begin{array}{l}0.05 \mathrm{ml} \text {. Contrôle TCA } 4 \text { heures plus tard } \\
\text { Selon les résultats du rapport TCAm/TCAt obtenus } 4 \text { heures plus tard, la conduite A, B ou } \\
\text { C a été adoptée }\end{array}$ \\
\hline
\end{tabular}

tée si nécessaire et remise en place. Lorsque le saignement était engendré par une rupture artérielle, le vaisseau a été suturé sous anesthésie locale. Devant un hématome, les signes cliniques de compression ont été recherchés.

\section{Analyse statistique}

Le nombre de sujets nécessaires à cette étude a été estimé à 119 pour chaque groupe, en prenant en compte une incidence de saignement postopératoire de $4 \%$ dans le groupe acénocoumarol, une différence minimale à mettre en évidence entre les deux groupes de $10 \%$, une puissance de $80 \%$ et un seuil de significativité de $5 \%$. Le logiciel EPI INFO version $6.04 \mathrm{cfr}$ a été utilisé. L'homogénéité entre les deux groupes a été testée, suivie par la présentation des caractéristiques des séances d'extraction et des résultats des différents contrôles de la coagulation. Nous avons ensuite comparé l'incidence du saignement entre les deux groupes, et présenté les résultats de la coagulation le jour de l'hémorragie. Les tests statistiques utilisés sont les tests basés sur l'écart réduit, le test de Student, le chi 2 et le test exact de Fisher. Le risque relatif en fonction du traitement anticoagulant a été calculé. Tous les résultats sont présentés avec leurs intervalles de confiance à $95 \%$ et un seuil de significativité de $5 \%$.

\section{RÉSULTATS \\ Caractéristiques des patients}

La population de l'étude se compose de 135 patients dans le groupe acénocoumarol, 133 dans le groupe héparine calcique. Les caractéristiques des malades sont détaillées dans le Tab. 4. Initialement, les deux groupes étaient comparables.

\section{Caractéristiques des séances d'extra- ctions dentaires}

Pour le groupe acénocoumarol et pour le groupe héparine calcique, le nombre total des séances d'extractions était respectivement de 229 et 208, les moyennes de $1.70 \pm 0.15$ [1-5] et $1.56 \pm 0.13$ [ 1 - 4] $(p=0.19)$. Le nombre de dents extraites / malade / séance était respectivement de $1.96 \pm 0.23[1$ - 8] 
Tableau 4 : Caractéristiques initiales de la population de l'étude Initial characteristics of the trial population.

\begin{tabular}{|c|c|c|c|c|}
\hline Caractéristiques & $\begin{array}{c}\text { Total } \\
(n=268)\end{array}$ & $\begin{array}{l}\text { Héparine calcique } \\
\qquad(n=133)\end{array}$ & $\begin{array}{l}\text { Acénocoumarol } \\
(n=135)\end{array}$ & $\mathbf{p}$ \\
\hline Age (années) & $49.63 \pm 1.57[16-79]$ & $48.92 \pm 2.24[21-79]$ & $50.32 \pm 2.12[16-79]$ & 0.37 \\
\hline Sexe Hommes / Femmes & 156/112 (58.2 / 41.8) & $81 / 52$ (60.9 / 39.1) & $75 / 60 \quad(55.5 / 44.4)$ & 0.37 \\
\hline $\begin{array}{l}\text { Pathologies cardiovasculaires } \\
\text { Prothèses valvulaires } \\
\text { Valvulopathies } \\
\text { rhumatismales } \\
\text { Cardiopathies ischémiques } \\
\text { Autres * }\end{array}$ & $\begin{array}{ll}114 & (42.5) \\
76 & (28.3) \\
35 & (13.1) \\
43 & (16.0)\end{array}$ & $\begin{array}{ll}61 & (45.9) \\
34 & (25.6) \\
14 & (10.5) \\
24 & (18.0)\end{array}$ & $\begin{array}{ll}53 & (39.2) \\
42 & (31.1) \\
21 & (15.5) \\
19 & (14.1)\end{array}$ & 0.34 \\
\hline $\begin{array}{l}\text { Stades fonctionnels NYHA } \\
\text { I - II : insuffisance cardiaque - } \\
\text { III - IV : insuffisance } \\
\text { cardiaque + }\end{array}$ & $\begin{array}{cc}205 & (76.5) \\
63 & (23.5)\end{array}$ & $\begin{array}{l}105 \text { (78.9) } \\
28(20.1)\end{array}$ & $\begin{array}{ll}100 & (74.1) \\
35 & (25.9)\end{array}$ & 0.35 \\
\hline $\begin{array}{l}\text { Risque d'endocardite } \\
\text { infectieuse } \\
\text { Important } \\
\text { Modéré } \\
\text { Minime }\end{array}$ & $\begin{array}{ll}114 & (42.5) \\
88 & (32.8) \\
66 & (24.6)\end{array}$ & $\begin{array}{ll}61 & (45.9) \\
40 & (30.1) \\
32 & (24.1)\end{array}$ & $\begin{array}{ll}53 & (39.2) \\
48 & (35.5) \\
34 & (25.2)\end{array}$ & 0.71 \\
\hline $\begin{array}{l}\text { Electrocardiogramme } \\
\text { Rythme sinusal } \\
\text { Fibrillation auriculaire } \\
\text { Autres troubles du rythme }\end{array}$ & $\begin{aligned} 116 & (43.3) \\
136 & (50.7) \\
16 & (6.0)\end{aligned}$ & $\begin{aligned} 58 & (43.6) \\
66 & (49.6) \\
9 & (6.8)\end{aligned}$ & $\begin{aligned} 58 & (43.0) \\
70 & (51.8) \\
7 & (5.2)\end{aligned}$ & 0.84 \\
\hline $\begin{array}{l}\text { Hygiène bucco-dentaire } \\
\text { Bonne } \\
\text { Mauvaise }\end{array}$ & $\begin{aligned} 16 & (6.0) \\
252 & (94.0)\end{aligned}$ & $\begin{array}{cl}7 & (5.3) \\
126 & (94.7)\end{array}$ & $\begin{array}{cl}9 & (6.7) \\
126 & (93.3)\end{array}$ & 0.63 \\
\hline
\end{tabular}

NYHA : New York Heart Association

*Autres cardiopathies : cardiopathie hypertensive 13 cas, cardiomyopathie dilatée 5 cas, maladie thromboembolique veineuse 5 cas, fibrillation auriculaire isolée 5 cas, communication interauriculaire 5 cas, myxome auriculaire gauche 2 cas, insuffisance mitrale dystrophique 2 cas, cardiomyopathie hypertrophique 2 cas, cardiothyréose 2 cas, rétrécissement aortique congénital 1 cas, rétrécissement aortique dégénératif 1 cas, hypertension artérielle pulmonaire primitive 1 cas.

** Selon la classification de la Société de Pathologie infectieuse de langue française (SPILF) 1992 [4]

Les données sont exprimées en effectifs (pourcentages) et moyennes \pm intervalle de confiance $95 \%$ [extrêmes]

et $1.92 \pm 0.22[1-7](p=0.80)$. Pour les caractéristiques odontologiques et les modalités de réalisation des extractions, le Tab. 5 montre que les séances étaient comparables dans les 2 groupes, sauf pour le siège de l'intervention : les avulsions de dents inférieures ont été plus fréquentes chez les malades sous héparine calcique $(p=0.02)$.

\section{Résultats des contrôles de la coagu- lation}

Dans le groupe acénocoumarol, à $\mathrm{J}-1$, l'INR était entre 3.00 - 4.50 dans $60.7 \%$ des cas. A J0, I'INR pré-opératoire était dans les limites thérapeu- tiques (2.00 - 4.50) dans $79.1 \%$ des cas, et il était supérieur à 4.50 dans $14.4 \%$ des cas (Tab. 6). Dans le groupe héparine calcique, le TCA (zone thérapeutique retenue $=1.50-2.50$ ) et I'INR étaient dans la zone thérapeutique dans seulement $28.9 \%$ et $27.9 \%$ des cas à J-1 et dans $19.7 \%$ et $4.4 \%$ des cas à J0 (Tab. 6).

\section{Complications hémorragiques}

L'incidence des complications hémorragiques a été significativement plus élevée dans le groupe héparine calcique : $15.8 \%$ contre $6.7 \%(p=0.02$, $\mathrm{RR}=2.40$ ) selon l'effectif des patients, et $11.1 \%$ \begin{tabular}{l}
$\begin{array}{l}\text { médecine } \\
\text { buccale } \\
\text { chirurgie } \\
\text { buccale }\end{array}$ \\
\hline VOL. 15, N ${ }^{\circ} 2$ \\
2009 \\
\hline page 69
\end{tabular} 
Tableau 5 : Caractéristiques des séances d'extractions dentaires.

Characteristics of dental extractions sessions.

\begin{tabular}{|c|c|c|c|c|}
\hline Caractéristiques & $\begin{array}{c}\text { Total } \\
(n=437)\end{array}$ & $\begin{array}{l}\text { Héparine calcique } \\
\qquad(n=208)\end{array}$ & $\begin{array}{l}\text { Acénocoumarol } \\
(\mathbf{n}=229)\end{array}$ & $\mathbf{p}$ \\
\hline Nombre de dents extraites & $3.13 \pm 0.30[1-16]$ & $3.00 \pm 12.39[1-14]$ & $3.27 \pm 14.27[1-16]$ & 0.36 \\
\hline Site des extractions & & & & 0.64 \\
\hline Antérieur & $154(35.2)$ & $78(37.5)$ & 76 (33.2) & \\
\hline Postérieur ${ }^{* *}$ & $216(49.4)$ & $99(47.6)$ & $117(51.1)$ & \\
\hline Antérieur + postérieur & $67(15.4)$ & $31(14.9)$ & $36(15.7)$ & \\
\hline Types de gouttières & & & & 0.32 \\
\hline Extemporanées & 377 (86.3) & $183(88.0)$ & $194(84.7)$ & \\
\hline Résine & $60 \quad(13.7)$ & $25(12.0)$ & $35(15.3)$ & \\
\hline Siège des extractions & & & & 0.02 \\
\hline Maxillaire & 236 (54) & $124(59.6)$ & $112(48.9)$ & \\
\hline Mandibule & $201(46)$ & $84(40.4)$ & $117(51.1)$ & \\
\hline Antibioprophylaxie de l'El & & & & 0.48 \\
\hline Clindamycine & $133(30.4)$ & 69 (33.2) & $64(27.9)$ & \\
\hline Pristinamycine & $125(28.8)$ & $52(25.0)$ & $73(31.9)$ & \\
\hline Amoxicilline & $69(15.8)$ & $36(17.3)$ & $33(14.4)$ & \\
\hline Ampicilline+gentamycine ${ }^{\cdots *}$ & $3(0.0)$ & $1(0.0)$ & $2(0.0)$ & \\
\hline Ampicilline & $1 \quad(0.0)$ & & $1 \quad(0.0)$ & \\
\hline Pas d'antibioprophylaxie & $106(24.2)$ & $50(24.0)$ & $56(24.4)$ & \\
\hline
\end{tabular}

* Dents de 14 à 24 et de 34 à 44

** Dents de 15 à 18 , de 25 à 28 , de 35 à 38 et de 45 à 48

VOL. $15, \mathrm{~N}^{\circ} 2$ 2009

*** Malades sous antibiotiques par voie parentérale pour endocardite infectieuse active lors de l'inclusion dans l'étude

Les données sont exprimées en effectifs (pourcentages) et moyennes + intervalle de confiance 95 \% [extrêmes]

Tableau 6 : Résultats des contrôles de la coagulation pré-opératoires.

Results of pre-operative coagulation controls.

\begin{tabular}{|c|c|c|c|c|}
\hline & \multicolumn{2}{|c|}{ Groupe héparine calcique $(n=208)$} & \multicolumn{2}{|c|}{ Groupe acénocoumarol $(n=229)$} \\
\hline & $\mathrm{J}-1$ & J 0 & J -1 & J 0 \\
\hline INR & $1.78 \pm 0.07[1.04-4.00]$ & $1.40 \pm 0.04[0.97-3.40]$ & $3.30 \pm 0.11(2.00-4.50)$ & $3.40 \pm 0.16(1.32-8.69)$ \\
\hline$<2$ & $150(72.1)$ & $19(95.7)$ & - & $15(6.7)$ \\
\hline $2-2.99$ & $51(24.5)$ & $8(3.9)$ & $90(39.3)$ & $84(36.7)$ \\
\hline $3-4.50$ & $7(3.4)$ & $1(0.5)$ & $139(60.7)$ & $97(42.4)$ \\
\hline$>4.50$ & - & - & - & $33(14.4)$ \\
\hline TCAm/TCAt & $1.67 \pm 0.10[1.00-5.00]$ & $1.45 \pm 0.10[1.00-5.00]$ & $1.32 \pm 0.04[0.73-4.00]$ & $1.32 \pm 0.03[0.76-3.44]$ \\
\hline$<1.50$ & $121(58.2)$ & $153(73.6)$ & $182(79.5)$ & $186(81.8)$ \\
\hline $1.50-2.50$ & $60(28.9)$ & $41(19.7)$ & $46(20.1)$ & $42(18.3)$ \\
\hline $2.51-3.00$ & $12(5.8)$ & $4(1.9)$ & - & - \\
\hline$>3.00$ & $15(7.2)$ & $10(4.8)$ & $1(0.4)$ & $1(0.4)$ \\
\hline
\end{tabular}

INR : International Normalized Ratio ; analyse semi-automatique par méthode chronométrique (ISI des réactifs : 1.0 - 2.8).

TCA : Temps de Céphaline Activé ; analyse semi-automatique par méthode chronométrique.

TCAm /: TCAt : Temps de Céphaline Activé malade / Temps de Céphaline Activé témoin.

Les données sont exprimées en effectifs (pourcentages) et moyennes + intervalle de confiance à 95 \% [extrêmes] 
contre $3.9 \%(p=0.004, R R=2.80)$ selon le nombre des séances d'extractions (Tab. 7). Le siège de l'hémorragie était maxillaire dans $8.9 \%$ des cas, mandibulaire dans $5.2 \%$ des cas $(p=0.17)$. Les patients sous héparine ont saigné entre $\mathrm{J} 0$ et $\mathrm{J} 6$, ceux sous acénocoumarol entre J0 et J4. Le jour du saignement, l'INR était inférieur à 4.50 dans la plupart des cas dans le groupe acénocoumarol (Tab. 8). Chez les patients sous héparine calcique, l'INR était inférieur à 4.50 dans la majorité des cas, mais le rapport TCA malade / TCA témoin était supérieur à 2.50 dans $30.4 \%$ des cas (Tab. 8). Dans tous les cas, l'application des mesures d'hémostase locale a suffi pour contrôler le saignement.

\section{DISCUSSION Incidence des complications hémor- ragiques}

Dans cette étude, l'incidence des complications hémorragiques a été plus élevée dans le groupe héparine calcique. Le saignement postopératoire pouvait être précoce ou tardif (jusqu'à J6). Lors de la survenue d'hémorragie, le TCA du malade était supérieur à 2.50 fois le TCA du témoin dans $1 / 3$ des cas dans le groupe héparine, alors que la plupart des patients sous acénocoumarol présentaient un INR en deçà de la zone de surdosage. Enfin, les mesures d'hémostase locale ont été suffisantes pour traiter efficacement tous les cas de saignement.

Tableau 7 : Incidences des complications hémorragiques. Incidence of hemorrhagic complications.

\begin{tabular}{|c|c|c|c|c|}
\hline Saignement + & Héparine calcique & Acénocoumarol & $\mathbf{p}$ & $\mathbf{R R}$ \\
\hline $\begin{array}{l}\text { En fonction de l'effectif des patients } \\
\qquad \begin{array}{l}\text { n } \%) \\
\text { Intervalle de confiance } 95 \%\end{array}\end{array}$ & $\begin{array}{c}n=133 \\
21(15.8) \\
{[10.0-21.1]}\end{array}$ & $\begin{array}{c}n=135 \\
9(6.7) \\
{[3.1-12.3]}\end{array}$ & 0.02 & $\begin{array}{l}2.4 \\
{[1.1-5.0]}\end{array}$ \\
\hline $\begin{array}{l}\text { En fonction du nombre des séances } \\
n(\%) \\
\text { Intervalle de confiance à } 95 \%\end{array}$ & $\begin{array}{c}n=208 \\
23(11.1) \\
{[7.1-16.1]}\end{array}$ & $\begin{array}{c}n=229 \\
9(3.9) \\
{[1.9-7.1]}\end{array}$ & 0.004 & $\begin{array}{l}2.8 \\
{[1.3-5.9]}\end{array}$ \\
\hline
\end{tabular}

médecine buccale chirurgie buccale

VOL. $15, \mathrm{~N}^{\circ} 2$ 2009

page 71

Tableau 8 : Résultats de la coagulation le jour de l'hémorragie.

Coagulation results the day of hemorrhage.

\begin{tabular}{lcc}
\hline & Groupe héparine calcique $(\mathbf{n}=\mathbf{2 3})$ & Groupe acénocoumarol $(\mathbf{n}=\mathbf{9})$ \\
\hline INR & $2.22 \pm 0.42[1.06-4.96]$ & $3.55 \pm 0.80[1.95-5.86]$ \\
$<2.00$ & $13(56.5)$ & $1(11.1)$ \\
$2.00-2.99$ & $6(26.1)$ & $3(33.3)$ \\
$3.00-4.50$ & $3(13.0)$ & $3(33.3)$ \\
$>4.50$ & $1(4.3)$ & $2(22.2)$ \\
TCAm/TCAt & $0.34[1.03-4.00]$ & $1.50 \pm 0.12[1.15-1.76]$ \\
$<1.50$ & $7(30.4)$ & $3(33.3)$ \\
$1.50-2.50$ & $10(43.5)$ & $6(66.7)$ \\
$2.51-3.00$ & $3(13.0)$ & - \\
$>3.00$ & $3(13.0)$ & -
\end{tabular}

INR : International Normalized Ratio ; analyse semi-automatique par méthode chronométrique (ISI des réactifs : $1.0-2.8$ ).

TCA : Temps de Céphaline Activé ; analyse semi-automatique par méthode chronométrique.

TCAm /: TCAt : Temps de Céphaline Activé malade / Temps de Céphaline Activé témoin.

Les données sont exprimées en effectifs (pourcentages) et moyennes + intervalle de confiance à 95 \% [extrêmes] 
médecine

buccale

chirurgie

buccale

VOL. $15, \mathrm{~N}^{\circ} 2$ 2009

page 72
Chez les malades traités par AVK, afin de prévenir le saignement postopératoire des premières heures, la réalisation des extractions dentaires sous héparine est courante et présumée plus rassurante, car ce traitement est arrêté quelques heures avant l'acte et car sa demi-vie plus courte que celle des AVK ${ }^{[5-7]}$. Cependant, il est courant d'observer que l'hémorragie peut survenir plusieurs jours après une avulsion dentaire ${ }^{[8]}$, ce qui coïncide, chez les patients sous héparine, à la phase au cours de laquelle ils reçoivent à la fois des AVK et de l'héparine, et où le niveau de l'hypocoagulabilité est instable, exposant à la fois aux risques hémorragique et thromboembolique. De plus, dans cette étude, nous avons observé que le changement de l'anticoagulant et le relais par l'héparine avaient entraîné une déstabilisation de l'hypocoagulabilité déjà dés la phase préopératoire puisque les malades étaient en deçà de la zone thérapeutique dans $1 / 4$ des cas la veille des extractions et dans la majorité des cas le jour de l'intervention. Par ailleurs, ce protocole onéreux (hospitalisation de plusieurs jours, contrôles répétés de la coagulation) est en outre risqué : risque de $\mathrm{TIH}{ }^{[9]}$. On dispose de rares publications, incluant un nombre limité de patients sous héparine [5-7, 10-13], parmi lesquelles une étude algérienne, réalisée chez 30 patients sous héparine calcique, et qui rapporte un taux de saignement de $30 \%$ [13].

Contrairement aux autres types de chirurgie (chirurgie cérébrale, abdominale...), le saignement postopératoire dans la cavité buccale est facilement accessible aux dispositifs d'hémostase locale, qui ont démontré leur efficacité pour assurer une bonne hémostase immédiate et pour maîtriser une éventuelle hémorragie différée [7, 14-18]. Plusieurs études ont montré la possibilité d'effectuer les extractions dentaires en poursuivant les AVK aux doses thérapeutiques et en appliquant des mesures d'hémostase locale. Dans ces publications, le taux de saignement varie entre 1.6 et $16.6 \%$, et chez les patients qui ont saigné aucun décès ou séquelle n'a été rapporté [13-36]. Ces constatations sont à mettre en balance avec les décès rapportés après diminution ou arrêt temporaire des AVK pour des actes de chirurgie bucco-dentaire [1]. En conséquence, il apparaît que la poursuite d'un traitement AVK efficace permet d'effectuer les avulsions dentaires sans le recours à l'hospitalisation des patients, sans les exposer au risque thromboembolique, sans augmenter le risque hémorragique par rapport au relais par l'héparine, à condition d'assurer une bonne hémostase locale et une surveillance postopératoire par le chirurgien dentiste et par le médecin ou cardiologue traitant qui doit contrôler l'INR au cours de la semaine postopératoire. Récemment, des recommandations publiées par la Société Francophone de Médecine Buccale et Chirurgie Buccale en collaboration avec la Société Française de Cardiologie privilégient la pratique des extractions dentaires en poursuivant un traitement AVK efficace, avec un INR pré-opératoire allant jusqu'à 4, en utilisant les mesures locales d'hémostase (grade I A), Le recours au relais par I'héparine doit rester exceptionnel (classe II, niveau B) [37].

\section{Limites de l'étude}

Les problèmes liés à la disponibilité de l'héparine nous ont contraints à utiliser l'héparine calcique provenant de 3 laboratoires différents. Néanmoins, dans tous les cas, les posologies ont été adaptées aux résultats du TCA. En dépit de ces limites, cette étude a permis, pour la première fois, d'évaluer le risque hémorragique des extractions dentaires sous héparine auprès de 133 malades chez lesquels 208 séances d'extractions ont été pratiquées, alors que dans la littérature les données concernant cette évaluation restent peu connues ${ }^{[3]}$.

\section{CONCLUSION}

Les résultats de cet essai thérapeutique, comparant la poursuite de l'acénocoumarol au relais par l'héparine calcique en chirurgie buccale ont des conséquences cliniques et économiques très importantes. Ils montrent que le rapport bénéficerisque est en faveur de la poursuite de l'acénocoumarol et la réalisation des avulsions dentaires avec un INR pré-opératoire $\leq 4.50$. 


\section{RÉFÉRENCES}

1 - Wahl MJ. Dental surgery in anticoagulated patients. Arch Intern Med 1998 ; 158 : 1610-5.

2 - Madrid C. Doit-on modifier le traitement anticoagulant avant des extractions dentaires ? Rev Med Suisse $2005 ; 1: 1418-24$

3 - Prescrire Rédaction. Antivitamine K et extraction dentaire. Peu de risques avec un INR inférieur à 4. Rev Prescrire 2004 ; 253 : 615-8.

4 - Prophylaxie de l'endocardite infectieuse. Med Mal Inf $1992 ; 22: 119-41$.

5 - Roser SM, Rosenbloom B. Continuated anticoagulation in oral surgery procedures. Oral Surg Oral Med Oral Pathol $1975 ; 40: 448-57$.

6 - Mehra P, Cottrell DA, Bestgen SC, Booth DF. Management of heparin therapy in the high-risk, chronically anticoagulated, oral surgery patient: a review and a proposed nomogram. J Oral Maxillofac Surg $2000 ; 58: 198-202$.

7 - Souto JC, Oliver A, Zuazu-Jausoro I, Vives A, Fontcuberta J. Oral surgery in anticoagulated patients without reducing the dose of oral anticoagulant: a prospective randomized study. J Oral Maxillofac Surg 199 ; 32 : 27-54.

8 - Pélissier A, Pélissier-Gelé B. Hémorragies d'origine dentaires : les techniques locales d'hémostase. Act Odonto- Stomatol $1990 ; 170:$ 307-22.

9 - Aiach M, Boneu B, Patron G. Utilisation des héparines en pratique médicale courante. In : Recommandations du Groupe d'Etudes sur l'Hémostase et la Thrombose (GEHT). Utilisation des traitements antithrombotiques en pratique médicale courante. Sang Thromb Vaiss 1991 ; 5 ( nºspécial ) : 4-10.

10 - Todd DW, Roman A. Outpatient use of low-molecular weight heparin in an anticoagulated patient requiring oral surgery : case report. J Oral Maxillofac Surg 2001 ; $59: 1090-2$

11 - Johnson-Leong C, Robert E. The use of low-molecular-weight heparins in outpatient oral surgery for patients receiving anticoagulation therapy. J Am Dent Assoc $2002 ; 133: 1083-7$.

12 - Bloomer CR. Excessive hemorrhage after dental extractions using low-molecular-weight heparin (Lovenox ${ }^{\circledR}$ ) anticoagulation therapy. J Oral Maxillofac Surg 2004 ; $62: 101-3$.

13 - Boukaïs H. Apports et limites des techniques locales d'hémostase en chirurgie buccale chez les malades soumis aux anticoagulants. Thèse de Doctorat en Sciences médicales, Alger, 2004.

14 - Al Belazy FA, Amer MZ. Hemostatic effect of n-Butyl2-cyanoacrylate $\left(\right.$ Histoacryl ${ }^{\circledR}$ ) glue in warfarin-treated patients undergoing oral surgery. J Oral Maxillofac Surg 2003 ; $61: 1$ 405-9.

15 - Carter G, Goss A. Tranexamic acid mouthwash. A prospective randomized study of a 2- day regimen vs 5-day regimen to prevent postoperative bleeding in anticoa- gulated patients requiring dental extractions. Int J Maxillofac Surg $2003 ; 32$ : 504-7.

16 - Blinder D, Manor Y, Martinowitz U, Taicher S. Dental extractions in patients maintained on continued oral anticoagulant. Comparison of local hemostatic modalities. Oral Surg Oral Med Oral Path Oral Radiol Endod $1999 ; 88: 137-40$.

17 - Carter G, Goss A, Lloyd J, Tocchetti R. Tranexamic acid mouthwash versus autologous fibrin glue in patients taking warfarin undergoing extractions : a randomized prospective clinical study. J Oral Maxillofac Surg 2003; 61: 1432-5.

18 - Halfpenny W, Frazer JS, Adlam DM. Comparison of 2 hemostatic agents for the prevention of postextraction hemorrheage in patients on anticoagulants. Oral Surg Oral Med Oral Path Oral Radiol Endod 2001 ; $92: 257-9$.

19 - Borea G, Montebugnoli L, Capuzzi P, Magelli C. Tranexamic acid as a mouthwash in anticoagulant-treated patients undergoing oral surgery. Oral Surg Oral Med Oral Pathol $1993 ; 75$ : 29-31.

20 - Campbell JH, Alvarado F, Murray A. Anticoagulation and minor oral surgery : should be anticoagulation regimen be altered? J Oral Maxillofac Surg 2000 ; 58 : 131 5

21 - Martinowitz U, Mazar AL, Taicher S, Varon D, Gitel SN, Ramot B, Rakocz M. Dental extraction for patients on anticoagulant therapy. Oral Surg Oral Med Oral Pathol $1990 ; 70: 274-7$.

22 - Keiani Motlagh K, Loeb I, Legrang W, Daelemans P, Van Reck, J. Prévention des saignements potopératoires chez des patients sous anticoagulants. Effets de l'acide tranexamique. Rev Stomotol Chir Maxillofac 2003 ; 104 : 77-9.

23 - Della Valle A, Sammartino G, Marenzi G, Tia M, Espedito di Laura A, Ferrari F, Lo Muzio L. Prevention of postoperative bleeding in anticoagulated patients undergoing oral surgery: use of platelet-rich plasma gel. J Oral Maxillofac Surg 2003 ; 61 : 1275-8.

24 - Gaspar R, Brenner B, Ardekian L, Peled M, Laufer D. Use of tranexamic acid mouthwash to prevent postoperative bleeding in oral surgery patients on oral anticoagulant medication. Quintessence Int 1997 ; 28 : 375-9.

25 - Devani P, Lavery KM, Howell CJT. Dental extractions in patients on warfarin: is alteration of anticoagulant regime necessary? Br J Oral Maxillofac Surg 1998 ; 36 : 10711.

26 - Bodner L, Weinstein JM, Kleiner Baumderten. Efficacy of fibrin sealant in patients on various levels of oral anticoagulant undergoing oral surgery. Oral Surg Oral Med Oral Pathol Oral Radiol Oral Endod 1998 ; 86 : 421-4.

27 - Cannon PD, Dharmar VT. Minor oral surgical procedures in patients on oral anticoagulants- a controlled study. Aust Dent J 2003 ; 48 : 115-8.

\begin{tabular}{l}
$\begin{array}{l}\text { médecine } \\
\text { buccale } \\
\text { chirurgie } \\
\text { buccale }\end{array}$ \\
\hline VOL. 15, N ${ }^{\circ} 2$ \\
2009 \\
\hline page 73
\end{tabular}

page 73 
28 - Okada T, Futatsuki T, Takesaki H, Ooe T, Abe K. Postoperative bleeding after tooth extractions in patients controlled with warfarin : a clinico-statistical study on the factors influencing postoperative bleeding. Fukuaka Igaku Zasshi 2004 ; 95 : 218-23.

29 - Ramstrom G, Sindet-Pedersen S, Hall G, Blomback M, Alander U. Prevention of postsurgical bleeding in oral surgery using tranexamic acid without dose modification of oral anticoagulants. J Oral Maxillofac Surg 1993 ; 51:1211-6.

30 - Garcia-Darennes F, Darennes J, Freidel M, Breton P. Protocole d'adaptation des traitements aux antivitamines K avant extractions dentaires. Rev Stomatol Chir Maxillofac 2003 ; 104 : 69-72.

31 - Russo G, Dal Corso L, Biasiolo A, Berengo M, Pengo V. Simple and safe method to prepare patients with prosthetic heart valves for surgical dental procedures. Clin Appl Thrombosis Hemostasis 2000 ; 6 : 90-3.

32 - Evans IL, Sayers MS, Gibbons AJ, Price G, Snooks H, Sugar AW. Can warfarin be continued during extraction? Results of a randomized controlled trial. Br J Oral Maxillofac Surg 2002 ; 40 : 248-52.

33 - Barrero MV, Knezevic M, Martin MT, Llorente AV, Orengo Valverde JC, Jiménez FG, Pérez QL, Sarmiento SD, Diaz Cremades JM, Castellano Reyes JJ. Oral surgery in the patients undergoing oral anticoagulant therapy. Medicina Oral $2002 ; 7$ : 63-70.
34 - Blinder D, Manor Y, Martinowitz U, Taicher S. Dental extractions in patients maintained on oral anticoagulant therapy : comparison of INR value with occurrence of postoperative bleeding. Int J Oral Maxillofac Surg $2001 ; 30: 518-21$.

35 - Zanon E, Martinelli F, Bacci C, Cordioli GP, Girolami A. Safety of dental extraction among consecutive patients on oral anticoagulant treatment managed using a specific dental management protocol. Blood Coagul Fibrinolysis $2003 ; 14: 27-30$.

36 - Gaudy JF, Ankri A, Tager F, El Haddioui A, Bravetti P, Lafont A, Godly B. Anticoagulants and dental extractions. Arch Mal Cœur 2005 ; 98 : 859-66.

37 - Recommandations de la Société Francophone de Médecine Buccale et de chirurgie Buccale en collaboration avec la Société Française de Cardiologie. Prise en charge des patients sous traitement antivitamines $\mathrm{K}$ en chirurgie buccodentaire. Med Buc Chir Buc 2006 ; $12: 188-212$. buccale

chirurgie

buccale

VOL. $15, \mathrm{~N}^{\circ} 2$ 2009

page 74 